\section{POSTHOC: A FORTRAN program for conducting post hoc multiple comparisons among means}

\author{
RAY W. COOKSEY \\ Colorado State University, Fort Collins, Colorado 80523
}

POSTHOC is a FORTRAN program designed to conduct post hoc multiple comparisons (pairwise comparisons, nonpairwise comparisons, or both) among means of interest following analysis of variance. Such post hoc comparisons are useful in understanding the nature and patterns of cell mean differences implied by any significant omnibus $F$ tests reported by analysis of variance (Kirk, 1968; Winer, 1971). The program can perform any of seven types of multiple-comparison tests, depending upon the needs of the user. These seven tests include (1) the l.s.d. procedure, (2) Tukey's HSD procedure, (3) the Newman-Keuls procedure, (4) Duncan's new multiple range test, (5) Scheffé's procedure for all pairwise contrasts, (6) Scheffẻ's procedure for specified nonpairwise contrasts, and (7) Dunnett's test for comparing treatment groups with a control (one- or two-tailed tests permitted). All seven of these procedures have been thoroughly reviewed by Keppel (1973), Kirk (1968), and Winer (1971).

POSTHOC can deal with an unlimited number of sets of means (useful where multiple dependent measures have been obtained in an experiment). Means arising from virtually any analysis of variance design can be submitted to POSTHOC as long as the appropriate error terms can be specified. The user has complete freedom as to which post hoc procedures are to be carried out on each set of means. For instances in which unequal ns for means are present, POSTHOC employs the harmonic mean for all post hoc tests except the Scheffé and Dunnett tests.

Input. Input to the program for each set of means to be analyzed consists of (1) the number of means to be compared, (2) the number and types of post hoc comparisons to be conducted on the set of means, (3) the appropriate error term from analysis of variance to be used by the post hoc procedures, (4) a set of six-character labels for the means in the analysis, (5) the actual means to be compared, (6) the number of observations for each mean in the analysis, (7) the critical value or values (obtained from statistical tables in Kirk, 1968; Winer, 1971 ; or some other source) needed to conduct each post hoc comparison requested at the appropriate level of Type I error, and (8) a matrix of contrast weights to be applied to the means if Scheffe's nonpairwise procedure is desired. The input information described

Comments on an earlier draft of this paper by Ross J. Loomis are appreciated. above must appear for each set of means to be analyzed. For multiple dependent measures, the sets of input data can be stacked one after another for analysis in a single program execution.

Output. Output from POSTHOC includes a list of selected program parameters and input information, such as means and number of observations per mean (as a check for input accuracy). Any pairwise comparisons desired are performed first by showing the entire rank-ordered pairwise mean difference matrix, appropriate critical difference values, and a summary of which pairs of means are significantly different at the user's chosen level of Type I error. Any nonpairwise Scheffé tests and/or Dunnett's test, if selected, are performed following any pairwise tests by showing the appropriate sets of contrast weights (input by the user) or treatmentcontrol differences and reporting the results of the significance tests (appropriate critical difference values and significance of each individual contrast).

Computer and Language. Program POSTHOC is written in ANSI FORTRAN IV and runs on both the CYBER 171 and 172 computers under the NOS 1.1 operating system (changes to run on CDC machines under SCOPE operating systems would be very minor, if any). No special external functions or library routines are necessary. The program is externally documented in a user's guide written by the author.

Restrictions. The program can deal with up to 20 means in a single set for analysis. This is a restriction imposed by the extent of the statistical tables available for obtaining the critical values for various post hoc tests, but can be altered readily if needed. If the nonpairwise Scheffé procedure is selected, the maximum number of contrasts that can be handled in a single analysis is 10 . There is no internal limitation on the number of sets of means that can be analyzed, and up to seven post hoc tests can be conducted on any single set of means. The core memory requirement for POSTHOC is $38 \mathrm{~K}$ on the CYBER 172 , and the program executes in an average range of 2 to $10 \mathrm{sec}$ for multiple analyses.

Availability. A source listing, user's guide, and sample input and output can be obtained free of charge by writing Ray W. Cooksey, Department of Psychology, Colorado State University, Fort Collins, Colorado 80523.

\section{REFERENCES}

KEPPEL, G. Design and analysis: A researcher's handbook. Englewood Cliffs, N.J: Prentice-Hall, 1973.

KIRK, R. E. Experimental design: Procedures for the behavioral sciences. Beimont, Calif: Brooks/Cole, 1968.

WINER, B. J. Statistical principles in experimental design (2nd ed.). New York: McGraw-Hill, 1971.

(Received for publication September 12, 1979; revision accepted October $17,1979$. 